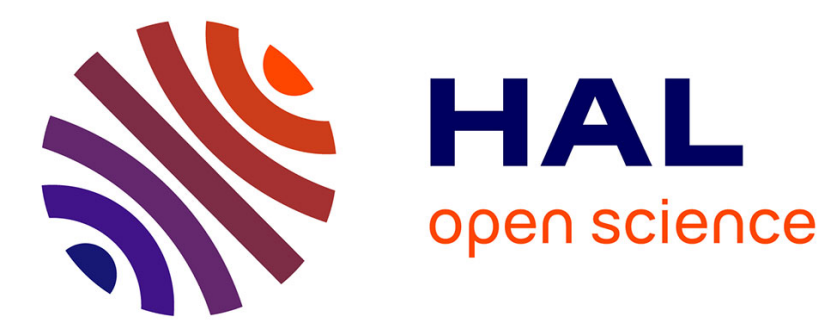

\title{
Model of a GaAs quantum dot embedded in a polymorph AlGaAs nanowire
}

Daniele Barettin, A. V. Platonov, A. Pecchia, V.N. Kats, G.E. Cirlin, A.D.

Bouravleuv, Lucien Besombes, Henri Mariette, M. auf Der Maur, A. Di Carlo

\section{- To cite this version:}

Daniele Barettin, A. V. Platonov, A. Pecchia, V.N. Kats, G.E. Cirlin, et al.. Model of a GaAs quantum dot embedded in a polymorph AlGaAs nanowire. IEEE Journal of Selected Topics in Quantum Electronics, 2013, 19 (5), pp.1901209. 10.1109/JSTQE.2013.2240657 . hal-00785275

\section{HAL Id: hal-00785275 \\ https://hal.science/hal-00785275}

Submitted on 5 Feb 2013

HAL is a multi-disciplinary open access archive for the deposit and dissemination of scientific research documents, whether they are published or not. The documents may come from teaching and research institutions in France or abroad, or from public or private research centers.
L'archive ouverte pluridisciplinaire HAL, est destinée au dépôt et à la diffusion de documents scientifiques de niveau recherche, publiés ou non, émanant des établissements d'enseignement et de recherche français ou étrangers, des laboratoires publics ou privés. 


\title{
Simulation of a GaAs quantum dot embedded into a AlGaAs nanowire
}

\author{
Daniele Barettin Department of Electronics Engineering, University of Rome Tor Vergata, Rome (Italy), A.V. \\ Platonov A.F.Ioffe Physical-Technical Institute, Politekhnicheskaya 26, 194021, St. Petersburg, Russia, Alessandro \\ Pecchia CNR-ISMN, via Salaria Km. 29.300, 00017 Monterotondo, Rome (Italy), V.N. Kats A.F.Ioffe \\ Physical-Technical Institute, Politekhnicheskaya 26, 194021, St. Petersburg, Russia, Spin Optics Laboratory, Saint \\ Petersburg State University, Ul'yanovskaya 1, Petrodvorets, St. Petersburg, 198904, Russia, G.E. Cirlin A.F.Ioffe \\ Physical-Technical Institute, Politekhnicheskaya 26, 194021, St. Petersburg, Russia, A.D. Bouravleuv A.F.Ioffe \\ Physical-Technical Institute, Politekhnicheskaya 26, 194021, St. Petersburg, Russia, L. Besombes CEA-CNRS \\ group "Nanophysique et Semiconducteurs", CEA, INAC, SP2M, and Institut Néel, 17 rue des Martyrs, F-38054 \\ Grenoble, France, H. Mariette CEA-CNRS group "Nanophysique et Semiconducteurs", CEA, INAC, SP2M, and \\ Institut Néel, 17 rue des Martyrs, F-38054 Grenoble, France, Matthias Auf der Maur Department of Electronics \\ Engineering, University of Rome Tor Vergata, Rome (Italy), Aldo Di Carlo Department of Electronics \\ Engineering, University of Rome Tor Vergata, Rome (Italy).
}

\begin{abstract}
We report on a numerical model of quasi onedimensional and quasi zero-dimensional semiconductor heterostructures. This model is strictly based on experimental structures of cylindrical nanocolumns of AlGaAs grown by molecular-beam epitaxy in the (111) direction. The nanocolumns are of $20-50 \mathrm{~nm}$ in diameter and $0.5-1 \mu \mathrm{m}$ in length and contain a single GaAs quantum dot, of $2 \mathrm{~nm}$ in thickness and 15 - $45 \mathrm{~nm}$ in diameter. Since the crystal phase of these nanowires spontaneously switches during the growth from zincblende to wurzite structures we implement a continuum elastic model and 8 band $\vec{k} \cdot \vec{p}$ model for polymorph crystal structures. The model is used to compute electromechanical fields, wave-function energies of the confined states and optical transitions. The model compares a pure zincblende structure with a polymorph in which the zincblende disk of GaAs is surrounded by wurtzite barriers and results are compared to experimental photoluminescence excitation spectra. The good agreement found between theory and features in the spectra supports the polyphorm model.
\end{abstract}

Index Terms-Nanowire, quantum dot, polymorph materials.

\section{INTRODUCTION}

Since the discovery of self-assembled quantum dots (QD), the field of optoelectronics has moved interest on nanoobjects of different dimension, size and shape in the search of novel useful properties. The challenge of the QD fabrication technology is to obtain highly homogeneous arrays with little dispersion in size, composition and shape in order to maximize quantum efficiency of LEDs and lasers. This requirement has been solved only partially with self-organization[1] that allows to achieve a rather uniform distribution of the dot sizes with dispersion of $10 \%$ [2]. However, the technique has a number of essential ineradicable lacks. For instance, because of the presence of a wetting layer, it is impossible to reach high quantum efficiencies. Furthermore, this growth process offer little to no control over the dot size, an essential parameter in order to tune the emission wavelength.

Daniele.Barettin@uniroma2.it
Thanks to the fast development and improvement of control in the growing conditions of nanostructures research has moved to new solutions in nano-engineering quantum confined states. The growth of free standing semiconductor nanowires (NW) and nanocolumns (NC) is one of the most promising solution for application in nanoelectronics [3], and nanophotonics [4] thanks to a better relaxation of the strain field that allows more freedom in combining III-V materials. The technology of manufacturing such NWs is now intensively developing [5]-[15]. These NWs can include one or several quantum dots (or quantum disks). Modern epitaxial methods allows to control the sizes and location of NW at the nanometer scale, that favorably distinguishes such QDs from planar and is very attractive for applications in optoelectronic devices.

However, GaAa/AlGaAs NWs also possess growth mechanisms that might have strong influences on their electronic properties. It is known [16] that the crystal structure of a $<111>$ nanowire may spontaneously switch during the growth from zinc-blende $(\mathrm{Zb})$ to wurzite $(\mathrm{Wz})$ phases. The effect leads to a strong modification of the time resolved photoluminescence spectra in the range of the optical transitions within the barrier material [13]. Similarly, it is expected that optical transitions in the QDs are sensitive to the crystal phase of the dot itself and its surrounding media. For example the energy position of the ground and excited states in the QD may be influenced by the electric field originating from the crystal phase transition. To observe the effect of this transition it is necessary to make a comparison between experimental data obtained by time resolved photoluminescence (PL) and photoluminescence excitation (PLE) measurements and theoretical simulations.

The electronic structures and the variation of the fundamental gap of the Wz phase of these crystals have been only quite recently studied. Knowledge of the full band structure of the these polymorph materials is fundamental in order to possibly extract parameters for tight-binding [18] (TB) and $\vec{k} \cdot \vec{p} \quad$ [17] model calculations. However, at present time only a relative 
small amount of parameters for bandstructure calculations are available in the literature.

Quasiparticle calculations of newly observed Wz polymorphs of InAs and GaAs has been reported in Ref.[19] using the GW approximation. De et al. have presented calculations of the bulk electronic band structures of non-nitride III-V semiconductors in the Wz phase using empirical pseudopotentials including spin-orbit coupling[20], while Jancu et al. have studied the electronic structure of the Wz phases of GaAs and InP using TB model and calculate the optical properties of crystal phase quantum dots formed by thin $\mathrm{Zb}$ layers inserted in a Wz-InP NW[21]. Since the Zb and Wz structures differ only by the arrangement of the second neighbors of a given atom, they used the same set of parameters for the two phases, while the atomic positions are given as input data and lattice constants are precisely determined by x-ray diffraction.

Very recently quasiparticle self-consistent $\mathrm{GW}$ calculations have been used to analyze the band structure of GaAs in Wz phase.[22]. Here, also the Kohn-Luttinger and Rashba-ShekaPikus effective Hamiltonian band structure parameters of Wz GaAs have been determined from these first-principles band structure calculations.

In this work, we have simulated an AlGaAs NW with an embedded GaAs QD, considering two different cases, a pure $\mathrm{Zb}$ case and a polymorph $\mathrm{Wz}-\mathrm{Zb}$ case, respectively. Dimensions, structural and geometric parameters have been strictly derived from experimental data. We have implemented an 8-band $\vec{k} \cdot \vec{p}$ model[29] for bandstructure calculations, and the parameters for the Pikus-Bir Hamiltonian of the Wz materials have been derived from the correspondent $\mathrm{Zb}$ materials using the cubic approximation as described by Chuang et al.[32], while the stiffness parameters of $\mathrm{Wz}$ crystals have estimated from Martin's transformations[27], [28] using the $\mathrm{Zb}$ parameters.

The paper is organized as follows. In the first section we present the experimental results that have inspired our numerical investigation. In section III we describe the models and the way we have derived all the parameters needed in the simulations, while all the numerical results are discussed in section IV. Finally, we give our conclusions.

\section{EXPERIMENT}

In these studies we have considered an AlGaAs NW with an embedded GaAs QD. AlGaAs NWs were grown on GaAs (111)B semi-insulating substrate using an EP1203 MBE system equipped with solid sources supplying $\mathrm{Ga}$ and $\mathrm{Al}$ atoms, and an As effusion cell to produce tetramers. The Al content along the NW and within the wire cross-sections was monitored by Raman spectroscopy in order to exclude possible inhomogeneities. We have found $\mathrm{Al}$ concentration in the range $c=0.24-0.26$. The growth was completed with deposition of GaAs to avoid possible oxidation of the AlGaAs shell layer. We did not perform any growth interruption at the heterointerfaces. The diameter of NWs in our samples is in the range 20-50 $\mathrm{nm}$. Scanning electron microscopy (SEM) and transmission electron microscopy (TEM) characterizations were performed before the optical measurements.
The samples were excited by either second harmonic of Nd:YAG laser or doubled Ti:Sa laser with 100 fs pulse duration and $70 \mathrm{MHz}$ frequency repetition. The average power of the lasers was about $10 \mu \mathrm{W}$, the spot diameter was 1.5 microns. Jobin-Yvon $30 \mathrm{~cm}$ monochromator and CCD detector were used for registration of the spectra. We used microscope to focus the laser beam and to collect the signal in the micro PL measurement of the single NW. The minimal diameter of the laser spot was 1.5 microns that allow us to extract the signal from a single NW. Additionally the set-up allows measure spectra with time resolution.

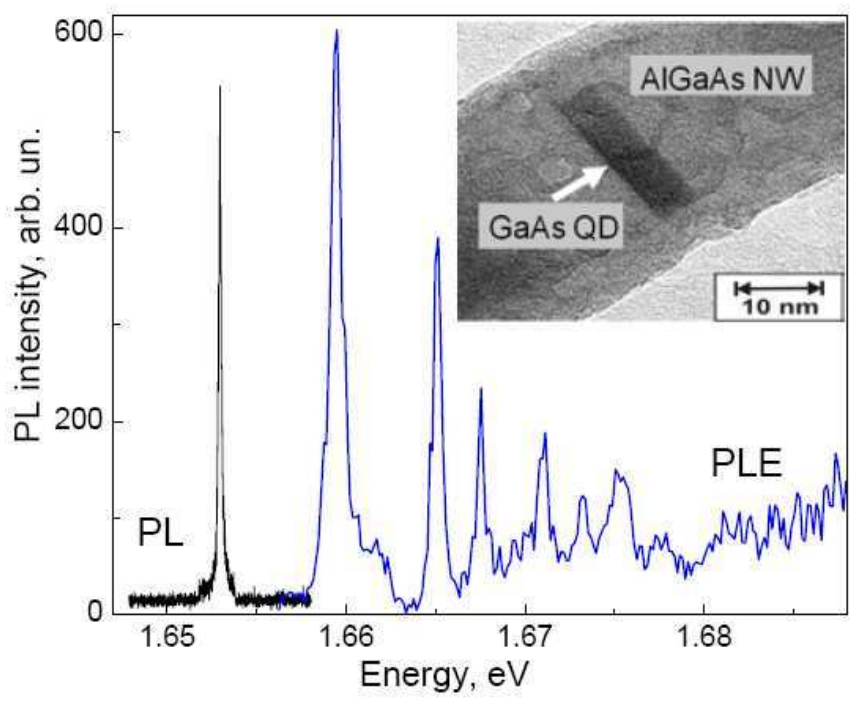

Figure 1. PL spectrum taken from a single QD at the excitation by $533 \mathrm{~nm}$ laser line with intensity of $0.1 \mathrm{~W} / \mathrm{cm}^{2}$. PLE spectrum taken from the same QD. Insert: TEM image of the GaAs QD in the AlGaAs NW.

The TEM image of the single AlGaAs NW with a slice of GaAs is shown in the insert of Figure 1. The dark area corresponds to the single GaAs QD. It is clearly seen that the interfaces along the axis of growth and along the radius are rather sharp. Considering cylindrical symmetry, the GaAs QD looks like a disk surrounded by the wide-gap semiconductor AlGaAs. The diameter of the NW in our samples was 25-50 $\mathrm{nm}$, the length $1000 \mathrm{~nm}$, and the thickness of the QD was of several mono-atomic layers, i.e., 2-5 nm.

Figure 1 shows PL spectra taken from a single QD embedded into a NW. At low excitation intensities of $\approx 1 \mathrm{~W} / \mathrm{cm}^{2}$ a single narrow line is observed at the energy of $1.6525 \mathrm{eV}$. The width of this line is $0.2 \mathrm{meV}$ and mainly limited by the spectral resolution of our spectrometer. It is natural to connect this line with the annihilation of an exciton in the single QD. This figure shows also the spectrum of photoluminescence excitation (PLE) taken from the same dot. The distance between the absorption (PLE) lines corresponds to the "transverse" carrier quantization in the dot of $15 \mathrm{~nm}$ in diameter. The energy of the "longitudinal" quantization is $\approx 130 \mathrm{meV}$, which corresponds to the QD thickness of $2.5 \mathrm{~nm}$. In the PLE spectrum one can see very broad absorption lines at high energy; we connect these lines with carrier absorption in the NW itself.

In Figure 2 a TEM image of one of our AlGaAs NW clearly shows the evidence of phase transitions from $\mathrm{Zb}$ to $\mathrm{Wz}$ crystal 


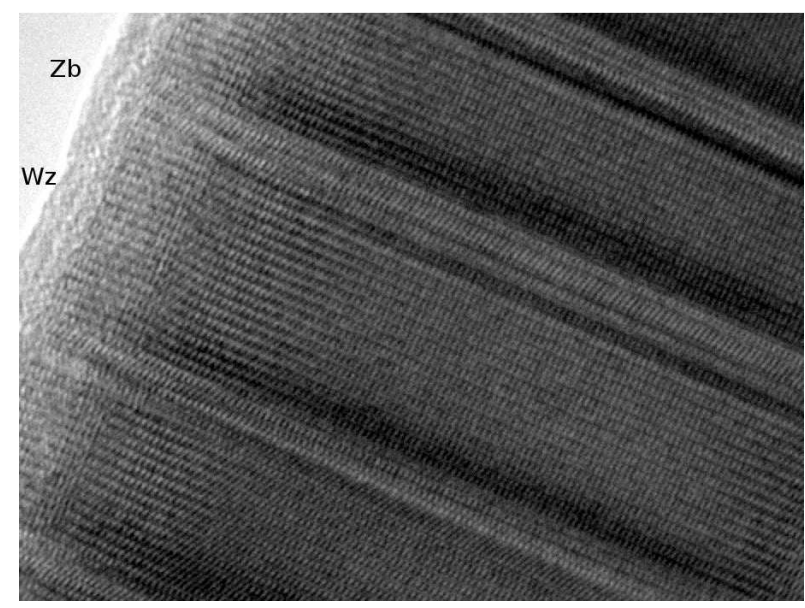

Figure 2. TEM image of an AlGaAs NW with wurtzites and zincblende phase alternations.

structures.

Here, layers in which the brighter lines apper parallel to the growth direction corresponds to $\mathrm{ABAB}$ lattice repetitions of $\mathrm{Wz}$, whereas the layers in which the bright lines appear to zigzag correspond to the $\mathrm{ABC}$ repetitions of the $\mathrm{Zb}$ phase. Interestingly, the darkest layers between the two crystal phases suggest the presence of a strain field given by a lattice mismatch between $\mathrm{Zb}$ and $\mathrm{Wz}$ AlGaAs.

\section{MOdelS}

\section{A. Strain calculations}

The continuum model we have used for our calculations of the electromechanical fields has been derived following the theory in Ref. [23]. The total mechanical and electrical free energy density change $d U$ of a piezoelectric medium can be written as[24]:

$$
d U=d U^{\text {mech }}+d U^{e l e c}=T d S+\sigma_{i k} d \varepsilon_{i k}+E_{i} d D_{i}
$$

where $T, S, \sigma_{i k}, \varepsilon_{i k}, E_{i}$, and $D_{i}$ are the temperature, entropy, stress tensor, strain tensor, electric field, and electric displacement, respectively.

With isentropic conditions $(d S=0)$ and using crystal symmetry considerations the elastic energy for a crystal with Zincblende (Zb) symmetry read [25]:

$$
\begin{aligned}
U_{Z b}^{\text {mech }} & =\frac{1}{2}\left[C_{11, Z b}\left(\varepsilon_{x x}^{2}+\varepsilon_{y y}^{2}+\varepsilon_{z z}^{2}\right)\right. \\
& +2 C_{12, Z b}\left(\varepsilon_{x x} \varepsilon_{y y}+\varepsilon_{x x} \varepsilon_{z z}+\varepsilon_{y y} \varepsilon_{z z}\right) \\
& \left.+4 C_{44, Z b}\left(\varepsilon_{x y}^{2}+\varepsilon_{x z}^{2}+\varepsilon_{y z}^{2}\right)\right]
\end{aligned}
$$

where $C_{i j, Z b}$ are the linearly independent stiffness constant parameters for a $\mathrm{Zb}$ structure.

Similarly, for a crystal with Wurtzite (Wz) symmetry we have for the elastic energy [25]:

$$
\begin{aligned}
U_{W z}^{\text {mech }} & =\frac{1}{2}\left[C_{11, W z}\left(\varepsilon_{x x}^{2}+\varepsilon_{y y}^{2}\right)+C_{33, W z} \varepsilon_{z z}^{2}\right. \\
& +2 C_{12, W z} \varepsilon_{x x} \varepsilon_{y y}+2 C_{13, W z} \varepsilon_{z z}\left(\varepsilon_{x x}+\varepsilon_{y y}\right) \\
& \left.+4 C_{44, W z}\left(\varepsilon_{x z}^{2}+\varepsilon_{y z}^{2}\right)+2\left(C_{11, W z}-C_{12, W z}\right) \varepsilon_{x y}^{2}\right]
\end{aligned}
$$

where $C_{i j, W z}$ are the linearly independent stiffness constant parameters for a $\mathrm{Wz}$ structure.

While the stiffness parameters for $\mathrm{Zb}$ Gas, AlAs and $\mathrm{AlGaAs}$ alloy can be found in literature[26], we do not have available the correspondent Wz parameters, so we have estimated them from Martin's transformations[27], [28] using the $\mathrm{Zb}$ parameters:

$$
\begin{aligned}
C_{11, W z} & =\frac{1}{6}\left(3 C_{11, Z b}+3 C_{12, Z b}+6 C_{44, Z b}\right) \\
& -3 \Delta^{2} /\left(C_{11, Z b}-C_{12, Z b}+C_{44, Z b}\right) \\
C_{12, W z} & =\frac{1}{6}\left(C_{11, Z b}+5 C_{12, Z b}-2 C_{44, Z b}\right) \\
& +3 \Delta^{2} /\left(C_{11, Z b}-C_{12, Z b}+C_{44, Z b}\right) \\
C_{13, W z} & =\frac{1}{6}\left(2 C_{11, Z b}+4 C_{12, Z b}-4 C_{44, Z b}\right) \\
C_{33, W z} & =\frac{1}{6}\left(2 C_{11, Z b}+4 C_{12, Z b}+8 C_{44, Z b}\right) \\
C_{44, W z} & =\frac{1}{6}\left(2 C_{11, Z b}-2 C_{12, Z b}+2 C_{44, Z b}\right) \\
& -6 \Delta^{2} /\left(C_{11, Z b}-C_{12, Z b}+4 C_{44, Z b}\right),
\end{aligned}
$$

where

$$
\Delta \equiv \frac{\sqrt{2}}{6}\left(C_{11, Z b}-C_{12, Z b}-2 C_{44, Z b}\right) .
$$

The piezoelectric field is included directly in our fullycoupled continuum model[23], and the not available Wz piezoelectric parameters have been approximated using parameters of well known Wz materials, as GaN, AlN and AlGaN alloy.

Expressions from 1 to 5 have been used to derive the governing equations of the electromechanical fields of the crystal[23].

\section{B. $\vec{k} \cdot \vec{p}$ method}

We have used for band structure calculations 8-band $\vec{k} \cdot \vec{p}$ model[29], which describes electron, heavy-hole, light-hole, and spin-orbit split-off bands around the $\Gamma$ point of the Brillouin zone and treats all other bands as remote bands. An $8 \times 8$ effective-mass Hamiltonian has been implemented, based on Foreman's application of Burt's exact envelope function theory to planar heterostructures [30].

The wave function of a state $n$ with energy $E_{n}$ is given by a linear combination of the eight Bloch parts weighted by the respective envelope functions,

$$
\psi_{n}(r)=\sum_{i=1}^{8} \phi_{i}(r) u_{i \Gamma}(r),
$$

where $\phi_{i}$ are the envelope function and $u_{i}$ are the Bloch states at $k=0$ [31]. 
The Luttinger-Kohn parameters the $\mathrm{Zb} \vec{k} \cdot \vec{p}$ Hamiltonian $\left(L_{1}, L_{2}, M_{1}, M_{2}, M_{3}, N_{1}\right.$, and $N_{2}$ ) have been taken from Ref.[26], while the $A_{i}$ parameters in the Pikus-Bir model for $\mathrm{Wz}$ have been derived from the correspondent $\mathrm{Zb}$ materials using the cubic approximation as described in Ref.[32]:

$$
\begin{array}{lrl}
A_{1}=-\gamma_{1}-4 \gamma_{2}, & A_{2}=-\gamma_{1}+2 \gamma_{2}, & A_{3}=6 \gamma_{2}, \\
A_{4}=-3 \gamma_{2}, & A_{5}=-3 \gamma_{3}, & A_{6}=-3 \sqrt{2} \gamma_{3},
\end{array}
$$

GaAs Pikus-Bir parameters are in a good agreement with the corresponding parameters given by $\mathrm{GW}$ calculations in Ref.[22]. For the band offset of the GaAs Wz material we refer to Ref.[16], and we have assumed an equivalent offset for the AlAs Wz material.

Results for electromechanical fields with the continuum model are included in the framework of the $\vec{k} \cdot \vec{p}$ as it is usually done via deformation potentials [33].

The complete set of parameters for the $\mathrm{Wz}$ materials we have used for our simulations is given in TableI, with the same

\begin{tabular}{|c|c|c|c|}
\hline \multicolumn{2}{|c|}{ Parameters } & GaAs Wz & $\overline{\mathrm{AlAs} \mathrm{Wz}}$ \\
\hline$a$ & $(\mathrm{~nm})$ & 0.399 & 0.4002 \\
\hline$c$ & $(\mathrm{~nm})$ & 0.653 & 0.654 \\
\hline$E_{g}$ & $(\mathrm{eV})$ & 1.552 & 3.216 \\
\hline$\Delta_{c r}$ & $(\mathrm{eV})$ & 0.010 & -0.169 \\
\hline$\Delta_{s o}$ & $(\mathrm{eV})$ & 0.017 & 0.019 \\
\hline$m_{e}^{\|}$ & $(\mathrm{eV})$ & 0.202 & 0.300 \\
\hline$m_{e}^{\perp}$ & $(\mathrm{eV})$ & 0.206 & 0.320 \\
\hline A & & -15.22 & -7.04 \\
\hline$A$ & 2 & -2.86 & -2.12 \\
\hline$A$ & 3 & 12.36 & 4.92 \\
\hline$A$ & 4 & -7.18 & -3.46 \\
\hline$A$ & 5 & -8.79 & -4.26 \\
\hline A & 6 & -12.43 & -6.02 \\
\hline$V B O$ & $(\mathrm{eV})$ & 0.716 & 1.246 \\
\hline$a_{1}$ & $(\mathrm{eV})$ & -6.8 & 1.0 \\
\hline$a_{2}$ & $(\mathrm{eV})$ & -8.6 & -7.2 \\
\hline$D_{1}$ & $(\mathrm{eV})$ & -3.7 & -17.1 \\
\hline$D_{2}$ & $(\mathrm{eV})$ & 4.5 & 7.9 \\
\hline$D_{3}$ & $(\mathrm{eV})$ & 8.2 & 8.8 \\
\hline$D_{4}$ & $(\mathrm{eV})$ & -4.1 & -3.9 \\
\hline$D_{5}$ & $(\mathrm{eV})$ & -4.0 & -3.4 \\
\hline$D_{6}$ & $(\mathrm{eV})$ & -5.5 & -3.4 \\
\hline$C_{11}$ & (GPa) & 145 & 141 \\
\hline$C_{12}$ & (GPa) & 51 & 49 \\
\hline$C_{13}$ & (GPa) & 38 & 41 \\
\hline$C_{33}$ & (GPa) & 158 & 149 \\
\hline$C_{44}$ & (GPa) & 38 & 40 \\
\hline$e_{13}$ & $\left(\mathrm{C} / \mathrm{m}^{2}\right)$ & -0.49 & -0.65 \\
\hline$e_{33}$ & $\left(\mathrm{C} / \mathrm{m}^{2}\right)$ & 0.81 & 1.50 \\
\hline$e_{15}$ & $\left(\mathrm{C} / \mathrm{m}^{2}\right)$ & -0.11 & -0.24 \\
\hline
\end{tabular}
usual notation as in Ref.[26].

Table I

PARAMETERS FOR THE WZ STRUCTURES USED IN THE SIMULATIONS.

Optical properties are calculated in the dipole approximation, starting from the dipole matrix elements $\vec{\mu}_{n m}$, between states $n$ and $m$, respectively. These are calculated from the momentum matrix elements which is represented in k-space as [34]

$$
p_{n m}=\frac{m_{0}}{\hbar} \frac{\partial H_{n m}}{\partial \vec{k}}
$$

Finally, oscillator strengths for the optical transitions are given by [36]:

$$
\vec{P}_{n m}=\frac{2 \pi e^{2} \hbar^{2}}{\epsilon_{0} m_{0}^{2} V}\left|\hat{e} \cdot \vec{p}_{n m}\right|^{2},
$$

where $\hat{e}$ is the direction unit vector of the electric field of the linearly polarized incident light, $V$ is the quantum dot volume, $e$ is the electron charge, and $\epsilon_{0}$ is the vacuum permittivity.

\section{Geometry and structure}

In our simulations we have considered an $\mathrm{Al}_{c} \mathrm{Ga}_{1-c} \mathrm{As}$ nanowire $(c=0.25)$ with a diameter of $40 \mathrm{~nm}$ and height of $50 \mathrm{~nm}$, with an embedded GaAs QD with a diameter of $20 \mathrm{~nm}$ and an hight of $5 \mathrm{~nm}$. Two different cases have been studied and compared: a pure $\mathrm{Zb}$ case, where the whole structure is assumed to be grown in pure $\mathrm{Zb}$ crystal phase, and a mixed structure as shown in Figure 3, where the GaAs QD is sandwiched by two adjacent $8 \mathrm{~nm}$ high $\mathrm{Wz}$ layers and surrounded by a $\mathrm{Zb}$ AlGaAs shell. The mixed structure is completed by $\mathrm{Zb}$ layers at the top and at the bottom.

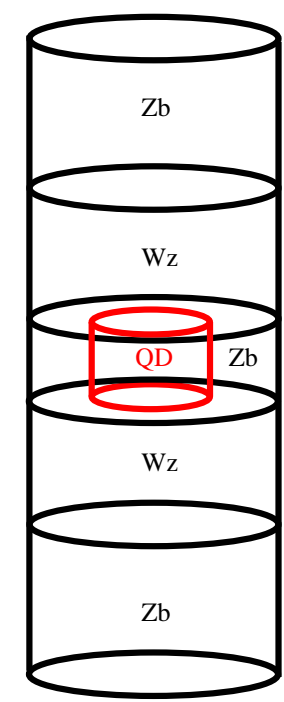

Figure 3. A scheme of the simulated AlGaAs nanowire with the embedded GaAs quantum dot (QD) for Mx case. The different crystal phases of the correspondent layers are also indicated. All the dimensions are given in the text.

We will refer in the following to this structure as the mixed (Mx) case.

\section{RESUlTS}

\section{A. Electromechanical fields}

The electromechanical fields (strain and piezoelectric fields) are evaluated as described in section III-A. In Figure 4 we plot the magnitude of the absolute value of the strain field for the pure $\mathrm{Zb}$ and the $\mathrm{Mx}$ structures, respectively.

It is interesting to notice that in the Mx case, besides the usual strain field inside and in the proximity of the QD, given by the lattice mismatch between the two materials, we can observe a weaker strain field at the interfaces between the two crystal phases of AlGaAs. This field is also observed in the TEM image shown in Figure 2. Despite its weakness, which 

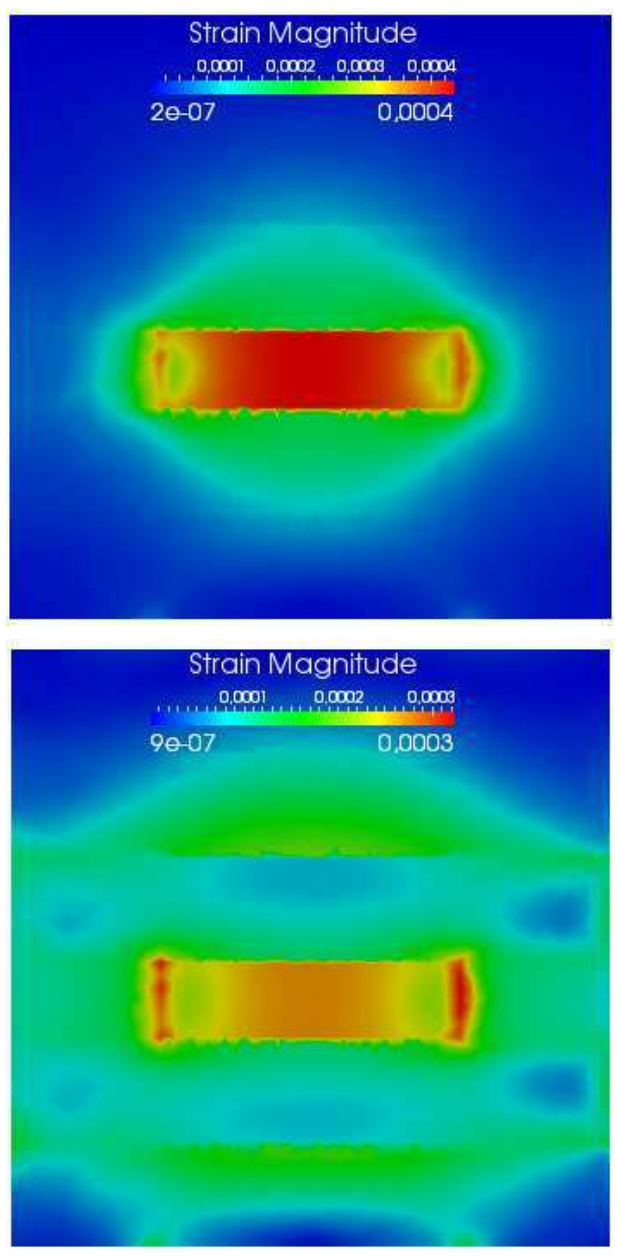

Figure 4. The magnitude of the absolute value of strain field for a pure $\mathrm{Zb}$ structure (top) and for the Mx structure (bottom).

was indeed expected from the small lattice mismatch between the GaAs and the $\mathrm{Al}_{c} \mathrm{Ga}_{1-c} \mathrm{As}(c=0.25)$ and the even smaller crystal mismatch between $\mathrm{Zb}$ - and $\mathrm{Wz}-\mathrm{AlGaAs}$, the strain field is non zero practically everywhere in the nanowire. This also affects the electric properties of the structure.

The electric field originating from the piezoelectric effect in the $\mathrm{Mx}$ case is relatively strong and presents an unusual shape, different from the typical results obtained for Zb QDs. This can be observed in Figure 5, showing the $z$ component of the electric field for both $\mathrm{Zb}$ and $\mathrm{Mx}$ structures.

Instead of the usual quadrupole shape obtained for $\mathrm{Zb}$ QDs, the Mx case gives a Wz-like shape of the electric field close and inside the dot, with its typical dipole characteristics, and opposite signs inside and just outside of the top and the bottom of the dot. This occurs even though the central volume of the column is in a pure $\mathrm{Zb}$ crystal phase and owes to the stronger magnitude of the piezoelectric effect induced by the Wz layers, which leads to stronger a electric field. A stronger magnitude of the piezoelectric effect in $\mathrm{Wz}$ heterostructures compared to $\mathrm{Zb}$ heterostructures has been already reported in the literature [25].
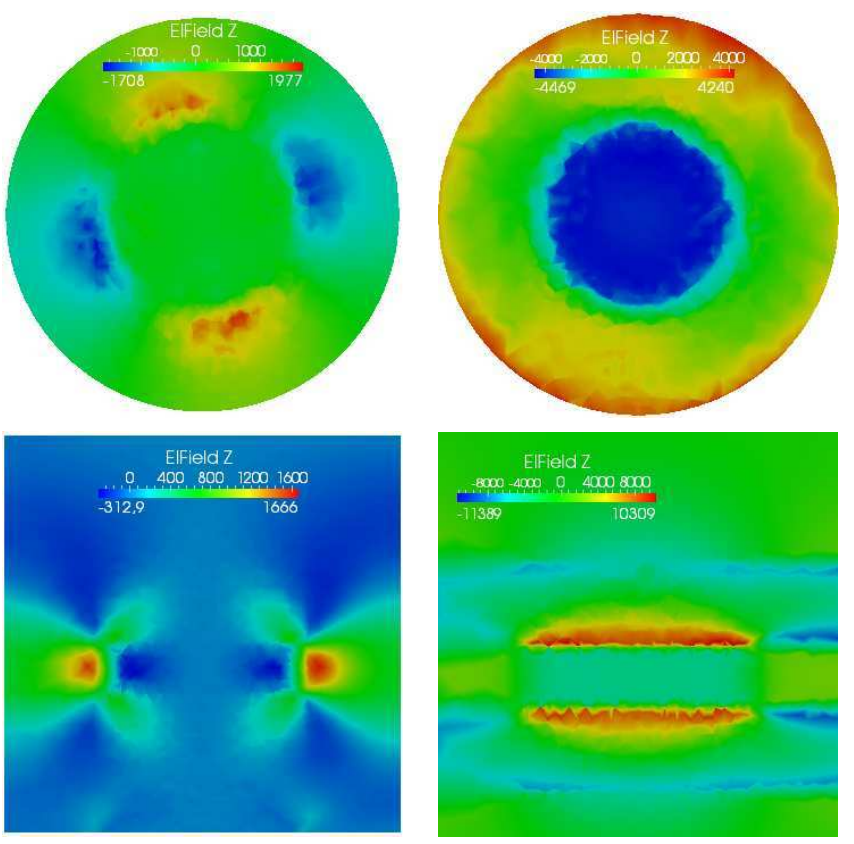

Figure 5. The $z$ component of the electric field in the $x y$ plane in the middle of the QD (top), and in the $x z$ planes (bottom), respectively. Left column $\mathrm{Zb}$ structure, right column Mx structure. All values in GVolt/m.

\section{B. Results for confined states}

The quantum confinement in the QD is mainly given by the band offset between the two materials and between the two different crystal structures of the AlGaAs in the Mx case, since the impact of the observed strain fields on the confinement via the deformation potentials[33] is not particularly relevant. In Figure 6 we plot the conduction $\left(E_{c}\right)$ and valence $\left(E_{v}\right)$ band along the $z$ direction for the considered structures, i.e., $\mathrm{Zb}$ and mixed.

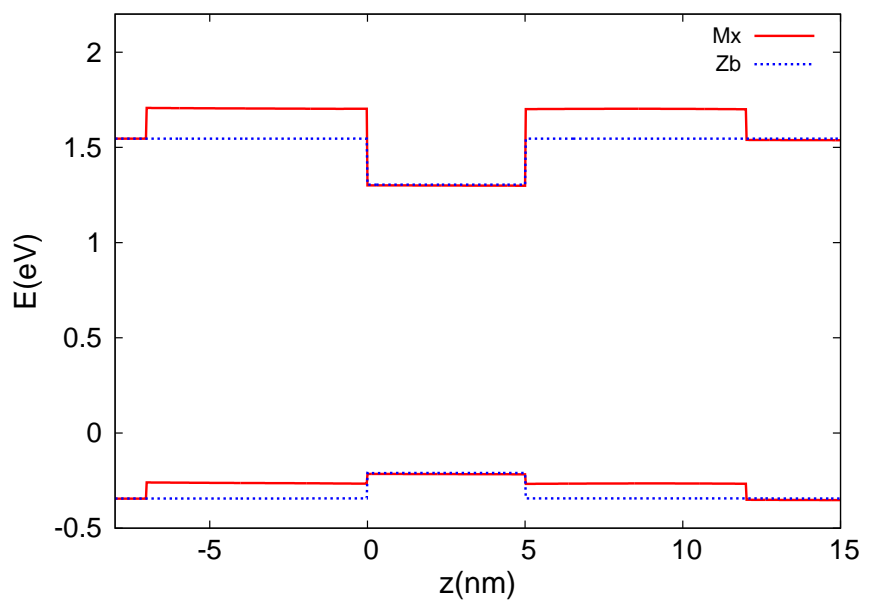

Figure 6. Conduction (top part of the figure) and valence (bottom part of the figure) band along the $z$ direction for both the $\mathrm{Zb}$ and the Mx cases The energy is expressed in $\mathrm{eV}$, where the 0 is given by the Fermi level.

The usual case of a $\mathrm{Zb}$ QD embedded in a $\mathrm{Zb}$ matrix has been considered as a useful comparison in order to better understand the results for the energy levels. For the $\mathrm{Mx}$ conduction band, the well of the QD is surrounded by the energetically higher barrier of the Wurtzite layers, higher as 
compared to the lower potentials of the $\mathrm{Zb}$ AlGaAs. This results in an increased quantum confinement for the electron states. On the other hand, the hole bands of the Mx structure exhibit a sort of double well with the potential profile of the QD and the two adjacent Wurtzite layers acting as a second wider well, that can contain several confined states. The form of the potential has a notable impact on the hole wave functions.

In Figure 7 we plot all the confined states for the electrons and the holes for both $\mathrm{Zb}$ and mixed situations. All states are doubly degenerate for spin inversion due to time reversal symmetry.

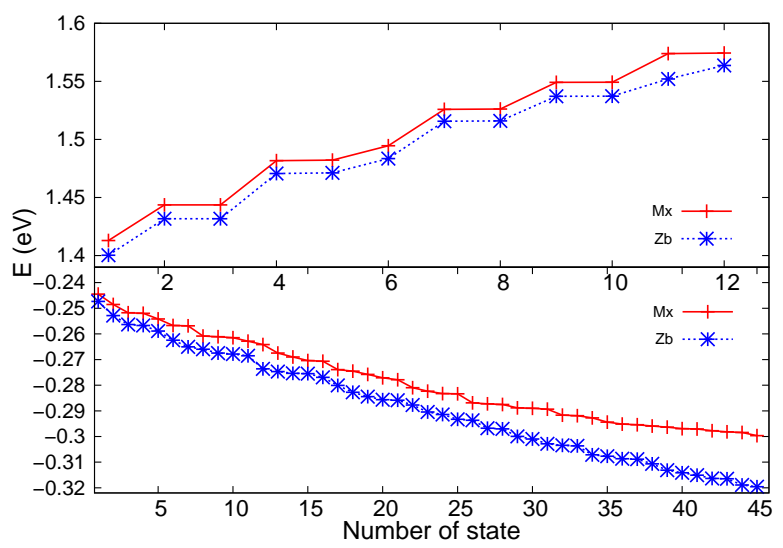

Figure 7. The calculated states for electrons (top) and holes (bottom). The $x$ scales enumerates the number of the correspondent state (different scale for electrons and holes), while the energy in the $y$ axis is referred to the Fermi level as in Figure 6.

As far as electron states are concerned, the main difference we observe between the $\mathrm{Zb}$ case and the Mx case is a higher degree of quantum confinement in the latter, which slightly increases the energy levels and gives raise to two further confined states, i.e., states 11 and 12 are delocalized in the $\mathrm{Zb}$ model, while confined within the QD in the Mx case.

The shape and the symmetry of the electron wave functions do not significantly differ between the two models, implying that they are mainly dependent on the ZB crystal structure of the QD, and are barely affected by the electric field and its symmetries. However, the actual lack of knowledge of the real values of piezoelectric coefficients for the $\mathrm{Wz}$ phase of these materials makes uncertain the impact of the electric field on the symmetry of the wave functions.

The higher electron states are completely delocalized in the $\mathrm{Zb}$ layers adjacent to the $\mathrm{Wz}$ layers. These states are not optically active, since they have almost zero overlap with the hole wave functions confined in the QD.

The situation for the hole states is notably different and quite interesting. Comparing the curves plotted in the bottom part of Figure 7, it is clearly possible to see that, while the first confined states of the two models are very close in energy (also showing a similar trend), higher energy states (in absolute value) display different trends. The states of the Mx model are systematically lower in energy. The onset of this change corresponds to states that start to delocalize in the Wz layers, adjacent to the QD. The increased available
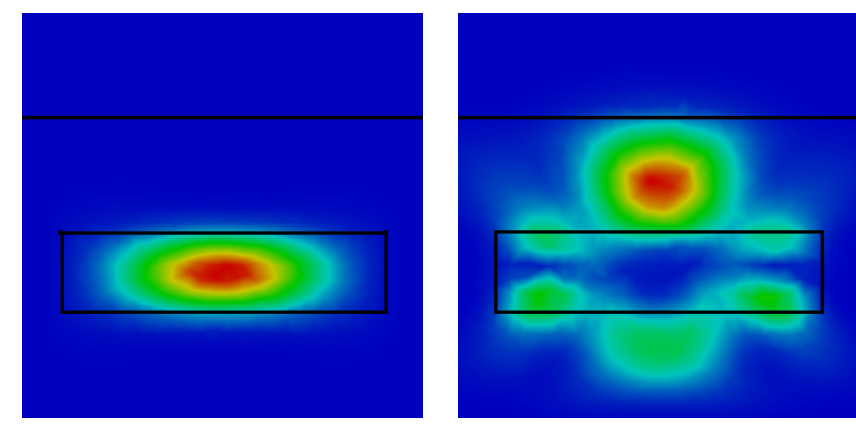

Figure 8. Holes ground state (left) and holes state number 32 according the bottom $y$ labeling of Figure 7 (right) in $x z$ plane $(x=30 \mathrm{~nm}$ and $z=40$ $\mathrm{nm}$ ). Continuous lines indicate the edges of the QD (below) and the edge between the $\mathrm{Wz}$ and the $\mathrm{Zb}$ phase (top).

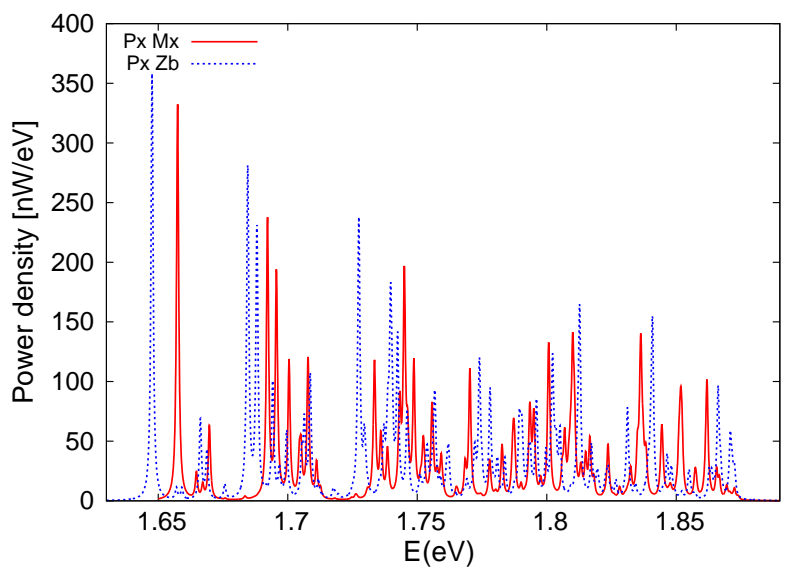

Figure 9. Oscillator strengths in $x$ directions for the two models.

volume explains their lower energy compared to the case of a pure $\mathrm{Zb}$ structure. This is confirmed by the contour plots of the probability density which we show in Figure 8 . Here we present a slice of the $x z$ plane, with $x=30 \mathrm{~nm}$ and $z=40$ $\mathrm{nm}$, showing basically the center of the nanowire with the dot and the two adjacent $\mathrm{Wz}$ layers.

Figure 8 (left) shows the probability density of the holes ground state, which, as expected, is completely confined in the QD. Figure 8 (right) shows the probability density of the hole state number 32, according the bottom $y$ labeling of Figure 7. This state is mainly confined in the Wz layers. A similar situation, with states confined in the volume formed by the QD and the two adjacent Wz layers, is observable for a considerable number of the higher energy hole states. All these states are optically active, since they have a non-zero overlap with the electron states confined in the dot.

\section{Oscillator strengths results}

We finally present results for the oscillator strengths, according dipole approximation. In Figure 9 we show the optical transitions.

Despite some resemblances in the distributions of the peaks, we can notice an effect which confirms what we have discussed in the previous section. We observe a blue shift of the lowest transitions of the $\mathrm{Wz}$ instance, namely for the ground state - ground state transition and the transitions between the 


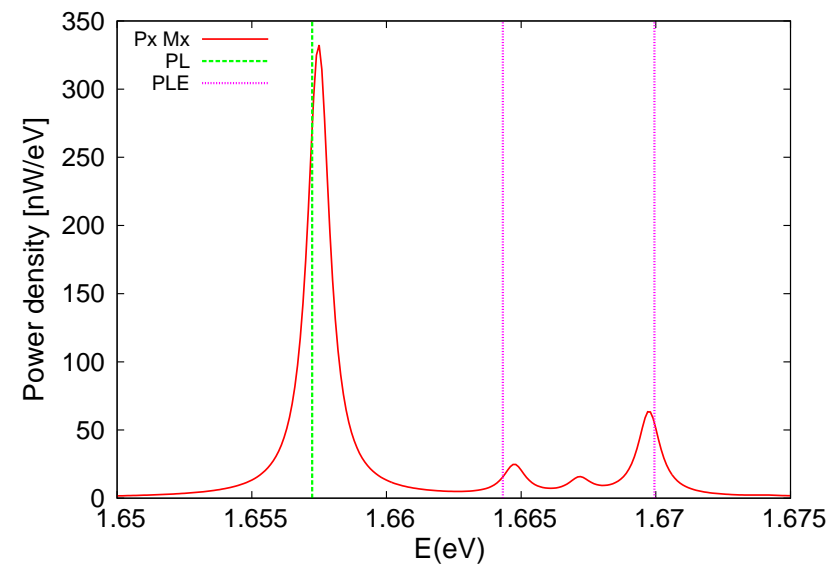

Figure 10. Oscillator strengths in $x$ directions for the Mx model compared with the positions of experimental transition results: PL for the first transition, PLE for the second and third ones.

more confined energy levels. This is clearly due to the higher energy of the electrons in the Mx case. But, on the other hand, for the peaks at higher energy we notice a red shift of the lines correspondent to the Mx models, due to the difference in energy between the less confined hole states, up to $20 \mathrm{meV}$ as observable in Figure 7.

Finally, in Figure 10, the lowest range of the spectrum is compared with the positions of three peaks given by experimental results from a specific nanowire sample with approximately the same dimensions of our model.

Here, the peak correspondent to the lowest transition, namely the ground state - ground state transition, has been measured by a PL experiment, while the other two positions we show are given by a PLE data set from the same sample. Although our Mx model cannot be completely validated due to the fluctuation of measurement data from different samples, the agreement for this particular sample is very good.

\section{CONCLUSION}

We have presented numerical simulations for polymorph Wz-Zb cylindrical AlGaAs NW with an embedded GaAs QD. Firstly, the connection with experimental structures has been shown, and consequently suitable continuum elastic and 8 band $\vec{k} \cdot \vec{p}$ models have been derived. All the necessary numerical parameters have been determined by appropriate approximations, where not available in the previous literature.

The results for the strain fields are in a good agreement with the experimental data, showing, besides the usual field given by the lattice mismatch between different material, a not-zero field at the interfaces between the two crystal phase of AlGaAs.

The analysis of the confined energy levels, wave functions and optical transitions clearly show the differences between an uniform crystal phase and a polymorph heterostructure. The comparison of numerical results and experimental spectra for optical transitions comfort the validity of our polymorph numerical model.

\section{REFERENCES}

[1] D. Leonard, K. Pond, P.M. Petroff, Phys.Rev. B50, 11687, (1994). 1

[2] J.-Y. Marzin, J.-M. Gerard, A. Izrael, D. Barrier, and G. Bastard, Phys.Rev.Lett. 73, 716, (1994). 1

[3] T. Bryllert, L.E. Wernersson, T. Lowgren, and L. Samuelson, Nanotechnology 17, S227 (2006). 1

[4] S. Gradecak, F. Qian, Y. Li, H.G. Park, and C.M. Lieber, Appl. Phys. Lett. 87, 173111 (2005). 1

[5] W. Seifert, M. Borgstrom, K. Deppert, K.A. Dick, J. Johansson, M.W. Larsson, T. Martensson, N. Skold, C.P.T. Svensson, B.A. Wacaser, L.R Wallenberg, and L. Samuelson, J.Cryst. Growth 272, 211 (2004). 1

[6] V.G. Dubrovskii, G.E. Cirlin, I.P. Soshnikov, A.A. Tonkikh, N.V. Sibirev, Yu.B. Samsonenko, and V.M. Ustinov, Phys. Rev. B71, 205325 (2005).

[7] J.C. Harmand, G. Patriarche, N. Péré-Laperne, M.-N. Mérat-Combes, L. Travers, and F. Glas, Appl. Phys. Lett. 87, 203101 (2005).

[8] M.C. Plante and R.R. La Pierre, J. Cryst. Growth 286 (2), 394 (2006).

[9] V.G. Dubrovskii, N.V. Sibirev, G.E. Cirlin, J.C. Harmand, and V.M. Ustinov, Phys. Rev. E 73, 021603 (2006).

[10] F. Glas and J.C. Harmand, Phys. Rev. B73, 155320 (2006).

[11] A.I. Persson, L.E. Fröberg, S. Jeppesen, M.T. Björk, and L. Samuelson, J. Appl. Phys. 101, 034313 (2007).

[12] V.G. Dubrovskii, N.V. Sibirev, G.E. Cirlin, A.D. Bouravleuv, Yu.B. Samsonenko, D.L. Dheeraj, H.L. Zhou, C. Sartel, J.C. Harmand, G. Patriarche, and F. Glas, Phys. Rev. B80, 205305 (2009).

[13] V.N. Kats, V.P. Kochereshko, A.V. Platonov, T.V. Chizhova, G.E. Cirlin, A.D. Bouravleuv, Yu.B. Samsonenko, I.P. Soshnikov, E.V. Ubyivovk, J. Bleuse, and H. Mariette, Semiconductor Science and Technology 27, 015009 (2012). 1

[14] G. Sallen, A. Tribu, T. Aichele, R. André, L. Besombes, C. Bougerol, M. Richard, S. Tatarenko, K. Kheng, and J.-Ph. Poizat, Phys. Rev. B84, 041405 (2011).

[15] Joël Bleuse, Julien Claudon, Megan Creasey, Nitin S. Malik, JeanMichel Gérard, Ivan Maksymov, Jean-Paul Hugonin, Philippe Lalanne, Phys.Rev.Lett. 106, 103601 (2011). 1

[16] D. Spirkoska,J. Arbiol, A. Gustafsson, S. Conesa-Boj, F. Glas, I. Zardo, M. Heigoldt, M. H. Gass, A. L. Bleloch, S. Estrade, M. Kaniber, J. Rossler, F. Peiro, J. R. Morante, G. Abstreiter, L. Samuelson, and A. Fontcuberta i Morrall, Phys. Rev. B 80, 245325 (2009). 1, 4

[17] J. M. Luttinger, W. Kohn, Phys. Rev. 97, 869 (1955).

[18] W.P. Harrison, Electronic Structure and the Properties of Solids, Dover Publications (1989)

[19] Z. Zanolli, F. Fuchs, J. Furthmüller, U. von Barth, and F. Bechstedt, Phys. Rev. B 75, 245121 (2007). 2

[20] A. De and Craig E. Pryor, Phys. Rev. B 81, 155210 (2010). 2

[21] J.-M. Jancu, K. Gauthron, L. Largeau, G. Patriarche, J.-C. Harmand, and P. Voisin, Appl. Phys. Lett. 97, 041910 (2010). 2

[22] Tawinan Cheiwchanchamnangij and Walter R. L. Lambrecht, Phys. Rev. B 84, 035203 (2011). 2, 4

[23] D. Barettin, S. Madsen, B. Lassen, and M. Willatzen, Commun. Comput. Phys., 11, 797-830 (2012). 3

[24] L.D. Landau and E.M.Lifshitz. Theory of Elasticity, Course of Theoretical Physics, Vol. 7, Pergamon Press (1970) 3

[25] V. A. Fonoberov and A. A. Balandin, J. Appl. Phys, 94, 7178 (2003). 3,5

[26] I. Vurgaftman, J.R. Meyer, L.R. and Ram-Mohan, J. Appl. Phys., Vol. 89,5815 (2001). 3, 4

[27] R. M. Martin, Phys. Rev. B 6, 4546 (1972). 2, 3

[28] A. F. Wright, J. Appl. Phys. 82, 6, (1997). 2, 3

[29] L. C. Lew Yan Voon and M. Willatzen, The $k \cdot p$ Method: Electronic Properties of Semiconductors, Springer (2009). 2, 3

[30] B. A. Foreman, Phys. Rev. B 48, 4964 (1993). 3

[31] E. P. Pokatilov and V. A. Fonoberov, V. M. Fomin and J. T. Devreese, Phys. Rev. B 64, 245328 (2001). 3

[32] S. L. Chuang and C. S. Chang, Phys. Rev. B, 54, 2491 (1996). 2, 4

[33] G. L. Bir and G. E. Pikus, Symmetry and Strain-Induced Effects in Semiconductors, Wiley New York, 295, 1974. 4, 5

[34] L.C. Lew Yan Voon and L.R. Ram-Mohan, Phys. Rev. B 47, 15500 (1993). 4

[35] A. Schliwa, M. Winkelnkemper, and D. Bimberg, Phys. Rev. B 76, 205324 (2007).

[36] O. Stier, M. Grundmann, and D. Bimberg, Phys. Rev. B 59, 5688 (1999). 4

\section{ACKNOWLEDGMENT}

The authors would like to thank... 


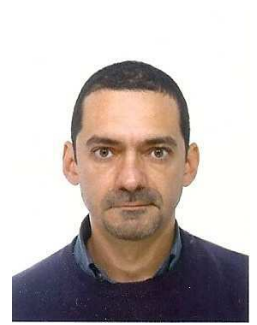

Daniele Barettin received the Master degree in Physics from the University of Rome "La Sapienza", Rome, Italy, and the Ph.D. degree in Applied Mathematical Models from the University of Southern Denmark. Since 2011 is Postdoctoral Researcher with the Department of Electronic Engineering of the University of Rome "Tor Vergata". He is been visiting researcher at the Ioffe Institute of Saint Petersburg, Russia. His research interests include the study of optoelectronic properties of nanostructures, analysis and optimization of organic electronic devices, and the study of complex systems in statistical mechanics.

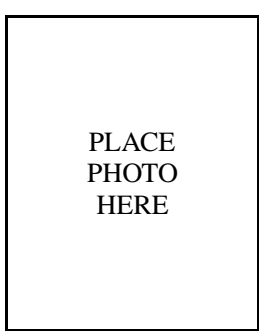

\section{A. Platonov}

\begin{tabular}{|l|}
\hline \\
PLACE \\
PHOTO \\
HERE \\
\end{tabular}

Alessandro Pecchia received the degree in physics from the University of Trieste, Trieste, Italy, in 1998 and the Ph.D. degree from The University of Leeds, Leeds, U.K., in 2002. He is a CNR researcher since 2005, working also in close collaboration with the Department of Electronic Engineering of the University of Rome Tor Vergata. His research is focused on transport properties of nanoscructures and on molecular electronics.

V.N. Kats

PLACE

PHOTO

HERE

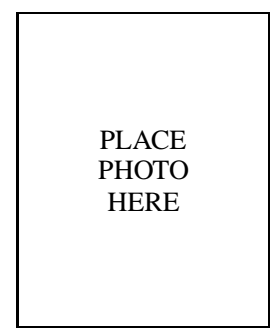

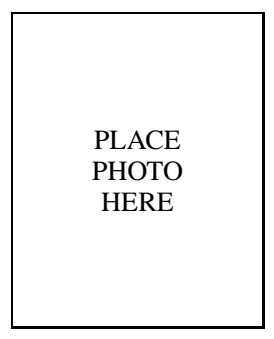

\section{A.D. Bouravleuv}

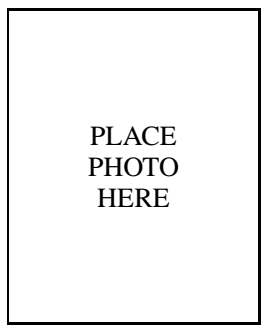

L. Besombes

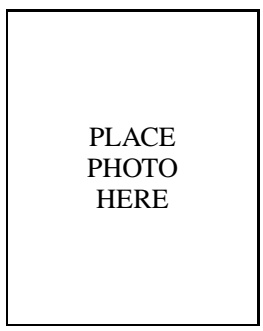

\section{H. Mariette}

PLACE

PHOTO

HERE

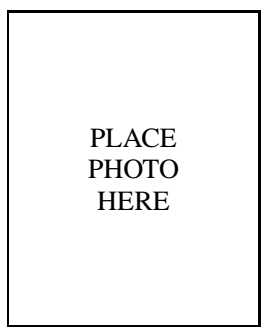

Aldo Di Carlo received the Laurea degree in physics (cum laude) from the University of Rome "La Sapienza", Rome, Italy, and the Ph.D. degree from the Technical University of Munich, Munich, Germany. In 1996, he became a Research Assistant in the Department of Electronic Engineering, University of Rome "Tor Vergata", Rome, where he is currently an Associate Professor, since November 2001 and a Coordinator of the Center for Hybrid and Organic Solar Energy. His current research interests include the study of electronic and optical properties of nanostructured devices, their analysis and optimization and the fabrication of organic electronic devices, the charge transport in nanostructured devices, high electron mobility transistors, organic thin-film transistors, molecular devices, and carbon nanotube-FETs. Dr. Di Carlo has been an Invited Speaker at the International Conference for the development of the nonequilibrium theory for the microscopic description of the transport process in nanostructured devices. 